\title{
What Does an Ionic Liquid Surface Really Look Like? Unprecedented Details from Molecular Simulations $\uparrow$
}

\author{
György Hantal, ${ }^{a, b}$ M. Natália D. S. Cordeiro ${ }^{b}$ and Miguel Jorge ${ }^{a, *}$ \\ Received (in $X X X, X X X) X$ th $X X X X X X X X X 20 X X$, Accepted $X$ th $X X X X X X X X X 20 X X$ \\ ${ }_{5}$ DOI: $10.1039 / \mathbf{b 0 0 0 0 0 0 x}$
}

We present the first intrinsic analysis of the surface of the [bmim] $\left[\mathrm{PF}_{6}\right]$ room-temperature ionic liquid. Our detailed analysis reveals unprecedented details about the structure of the interface by providing the relative prevalence of different 10 molecular orientations. These results suggest that experimental data should be reinterpreted considering a distribution of molecular arrangements.

Ionic liquids (ILs) have been heralded as the new green solvents and are finding more and more applications due to their ideal 15 physicochemical properties (e.g. non-volatility, non-flammability, stability over wide temperature ranges). Many applications involve processes that occur at the interface, meaning that their optimisation requires a profound knowledge of the true nature of that interface. Unfortunately, this has not yet been achieved, on 20 the one hand, because the accuracy and resolution of experimental methods are still insufficient to provide a detailed and coherent molecular-level description of IL surfaces, ${ }^{1}$ and, on the other hand, due to the limitations of standard simulation methods to probe the underlying structure of those surfaces. ${ }^{2} \mathrm{~A}$

25 major breakthrough in this context was the appearance of novel intrinsic sampling techniques that can clarify the true nature of fluid interfaces at the molecular level. ${ }^{2-7}$ In this communication we apply for the first time our intrinsic sampling techniques ${ }^{4-7}$ to a room-temperature ionic liquid. We chose 1-n-butyl-330 methylimidazolium hexafluorophosphate $\left([\mathrm{bmim}]\left[\mathrm{PF}_{6}\right]\right.$ ) (see Fig. 1), modelled by the force-field of Lopes et al., ${ }^{8}$ and carried out very long simulations ( $80 \mathrm{~ns}$ ) in a large system (512 ion pairs, with a surface of $5 \times 5 \mathrm{~nm}^{2}$ ) in order to obtain good statistics. Earlier simulation $^{9-12}$ and experimental ${ }^{1,13}$ results on $\left[\mathrm{bmim}^{\mathrm{b}}\right]\left[\mathrm{PF}_{6}\right]$ 35 have generated conflicting interpretations, but our intrinsic analysis reveals new details about the IL surface that help to clarify this controversy.

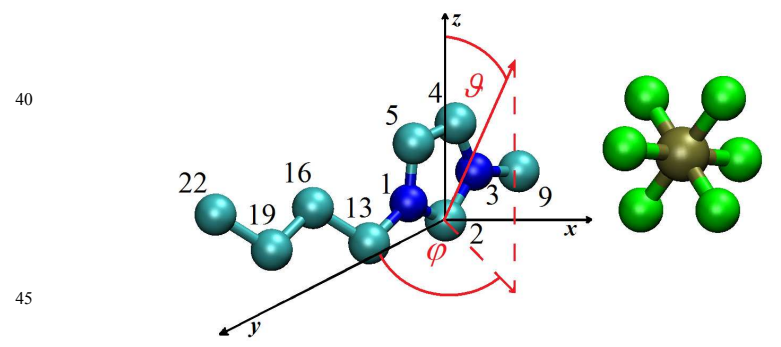

Fig. 1 Diagram depicting the $\mathrm{PF}_{6}$ anion and the bmim cation, with non-hydrogen atoms numbered, as well as the definition of the ion-fixed coordinate frame for orientation studies.

50

Fluid interfaces are notoriously difficult to analyse because, due to thermal fluctuations, their instantaneous position is constantly changing (contrary to solid interfaces, whose surface atom positions are fixed). Conventional analysis methods probe ${ }_{55}$ interfacial properties relative to a steady flat plane, which implies inevitable information loss as they cannot identify the interfacial molecules and determine the actual distance of the particles to the interface. ${ }^{2}$ However, this is extremely important when comparing simulation results to experiments that probe only interfacial 60 molecules. In contrast, intrinsic methods are able to decouple thermally activated capillary waves from the temperatureindependent intrinsic corrugation of the interface, and provide analysis of properties in terms of intrinsic distances. ${ }^{2-4}$ Our method (Fig. 2) is based on first identifying the surface atoms by ${ }_{65}$ probe spheres of a given size that are dropped from the vapor phase along grid lines perpendicular to the interface, ${ }^{5,6}$ After having identified the set of interfacial atoms one can construct a reference surface that passes through each surface atom. We have recently shown that using triangular interpolation is the most 70 efficient way to compute the position of the interface at any point. $^{7}$ The determination of the distance of each site in the system from the instantaneous surface (i.e. the intrinsic distance) makes it possible to compute the intrinsic profile of any property across the interface (further technical details are provided in 75 Supporting Information).
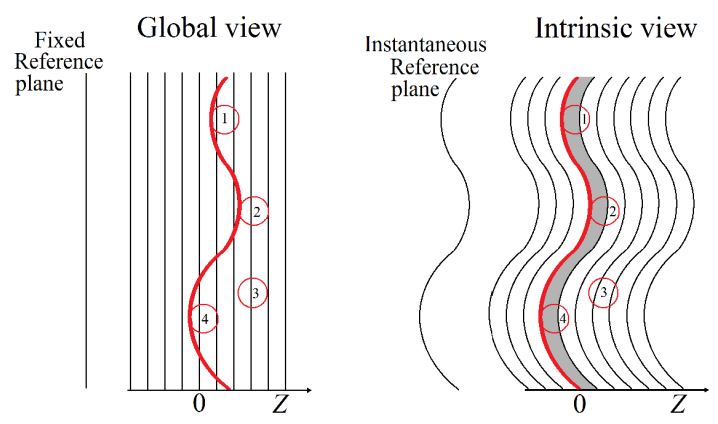

Fig. 2 Differences between the global (left-hand side) and intrinsic (right-hand side) view of the system. In the intrinsic view, contrary to the global view, particles 1,2 , and 4 are 80 identified as interfacial thus they contribute to the same bin of the density profile. 
Global and intrinsic density profiles of the system across the simulated interfaces are shown in Fig. 3. The global mass density in Fig. 3a shows a smooth density increase from the vapor to the liquid and only a slight density enhancement near the interface. 5 The intrinsic profile, however, (Fig. 3b) shows much sharper and higher density peaks close to the interface, revealing a strong molecular layering. Fig. 3c shows global density profiles of representative atoms ( $c f$. Fig. 1) that belong to interfacial IL molecules (a molecule is considered to be interfacial (i.e., it 10 belongs to the surface layer) if any of its atoms is detected by the intrinsic analysis technique ${ }^{5,6}$ ). The peaks for the different atoms follow each other in a consecutive order from $\mathrm{C} 22$ to $\mathrm{C} 9$, which illustrates that the cations are mainly oriented with their butyl chains toward the vapor phase. The anion is on average located 15 close to the N1 atom. The most interesting aspects of Fig. 3c, however, are the long tail of the $\mathrm{C} 22$ profile towards the liquid (marked with an arrow) and the shoulder of the C9 profile towards the vapor, which suggest more complex molecular arrangements at the surface. What are merely hints in the global 20 profiles arise as distinct features in the intrinsic profiles of Fig. $3 \mathrm{~d}$, since different molecular orientations give rise to clearly distinguishable peaks. For example, the C9 atom shows a sharp peak near the vapor, corresponding to atoms that are part of the actual IL surface, and a large peak in the direction of the liquid, 25 which corresponds to atoms that belong to interfacial molecules but that are further away from the actual surface.

\section{GLOBAL PROFILES INTRINSIC PROFILES}
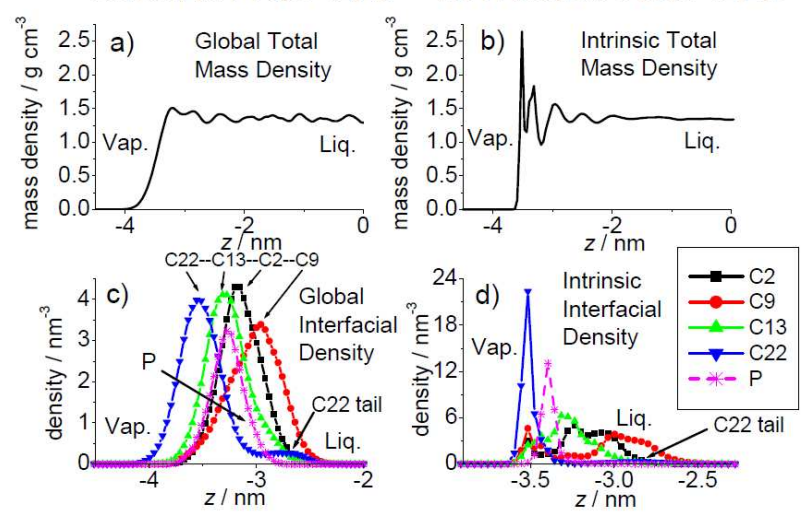

Fig. 3 Density profiles for the IL vapor-liquid interface. Panels a and $b$ show global and intrinsic mass density profiles, 30 respectively, in the total system. Panels c and d represent global and intrinsic number density profiles for atoms belonging to molecules found at the interface by our intrinsic method. ${ }^{4}$

A more complete picture can be obtained by performing a full 35 orientation analysis ${ }^{14}$ of the interfacial cations. The joint bivariate distribution of the polar angular coordinates of the surface normal $(P(\cos \vartheta, \varphi))$ can be determined in a molecule-fixed coordinate frame (see Fig. 1). The origin of this frame is fixed on the $\mathrm{C} 2$ atom; the $z$ axis is parallel with the symmetry axis of the 40 imidazolium ring, while the $y$ axis is parallel with the $\mathrm{NN}$ axis of the molecule. The $x$ axis is perpendicular to the ring plane. The information obtained from the orientation analysis of the bmim ring (shown in Fig. 4) can be completed by investigating the orientation of butyl chains - we calculated the distribution of the
${ }_{45}$ cosine of the angle $(\alpha)$ between the surface normal and the vector pointing from the first (C13) to the last (C22) carbon atom of the chain (Fig. 5). If we combine this detailed orientation analysis with the intrinsic profiles of Fig. 3d, we can obtain an unprecedented picture of the IL surface. The most important 50 molecular orientations identified in this study are shown in Fig. 6.
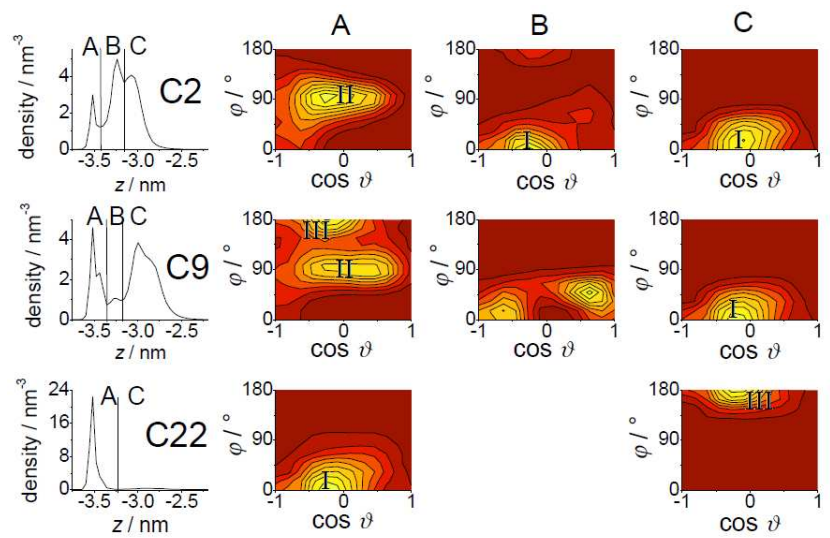

Fig. 4 Orientation maps corresponding to different regions of the intrinsic density profiles. Brighter tones represent higher probabilities. Three atoms, C2, C9 and C22, having the most 55 structured profiles were chosen for this analysis. The intrinsic distance range was divided at main minima separating maxima as illustrated in the first column. Regions toward the vapor and the bulk were denoted by A and C, respectively, while B refers to an intermediate region.

60

Orientation I (top of Fig. 6) is the most prevalent and appears in the maps of Fig. 4 as a peak with $\cos \vartheta=-0.15\left(\vartheta \approx 98^{\circ}\right)$ and $\varphi=0^{\circ}$. This corresponds to an arrangement in which the ring is normal to the surface with the N1-C13 bond almost precisely ${ }_{65}$ perpendicular. The peak for orientation I dominates the maps for zone $\mathrm{A}$ of the $\mathrm{C} 22$ profile, zone $\mathrm{C}$ of the $\mathrm{C} 9$ profile and zones $\mathrm{B}$ and $\mathrm{C}$ of the $\mathrm{C} 2$ profile. In these regions, the butyl chain is extended toward the vapor phase (circles in Fig. 5).

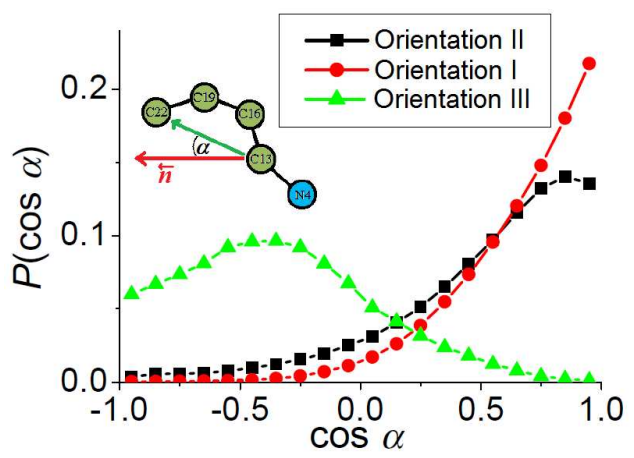

Fig. 5 Distribution of the cosine of the angle $(\alpha)$ between the surface normal and the vector pointing form the first to the last butyl carbon atom (see inset). The distribution is plotted for three regions dominated by each of the three preferred orientations: 75 orientation I was analyzed in region $\mathrm{C}$ of atom C9, orientation II in region $\mathrm{A}$ of atom $\mathrm{C} 2$ and orientation III in region $\mathrm{C}$ of atom C22 (see Fig. 4). 
The coupling of the intrinsic profiles with the orientation maps reveals two other important arrangements of surface cations. In orientation II (Fig. 6), the ring is oriented parallel to the surface giving rise to a peak at $\cos \vartheta=-0.27\left(\vartheta \approx 106^{\circ}\right)$ and $\varphi=90^{\circ}$. 5 This peak dominates the map for zone A of the $\mathrm{C} 2$ profile and also clearly appears in zone A of the C9 profile (Fig. 4). In this orientation, the butyl chain also prefers to extend toward the vapor phase (squares in Fig. 5). It should also be noted that orientation II exhibits a wide variability in values of $\cos \vartheta$, from 10 about -0.7 to about 0.8 , but a very narrow range of $\varphi$ values. This means that although on average the ring is parallel to the surface, it actually has great freedom to tilt along the $\mathrm{NN}$ vector.

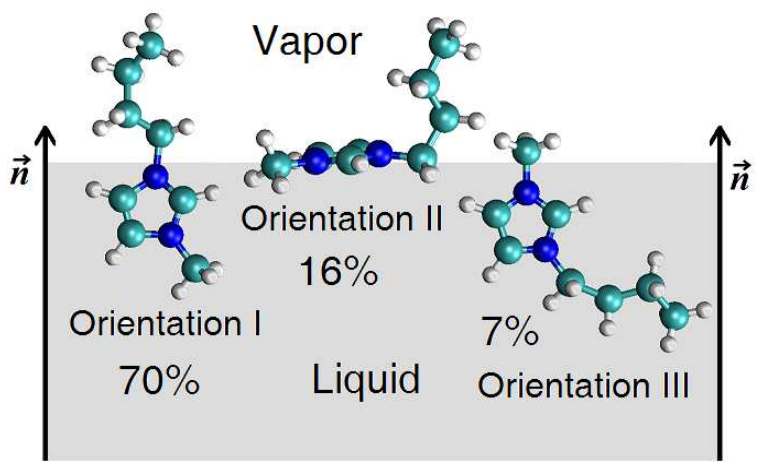

Fig. 6 The three most important molecular orientations of the 15 imidazolium cations found in our study. The relative prevalence of each typical orientation is also indicated.

Finally, we are able to identify a completely new arrangement, orientation III in Fig. 6, in which the ring is perpendicular to the 20 surface, the methyl group is pointing toward the vapor and the butyl chain is on the liquid side. This is manifested by a peak at $\cos \vartheta=-0.20\left(\vartheta \approx 102^{\circ}\right)$ and $\varphi=180^{\circ}$, which appears clearly in zone $\mathrm{C}$ of the $\mathrm{C} 22$ profile. It also appears in zone A of the C9 profile because these atoms are part of the surface layer, and in 25 zone $\mathrm{B}$ of the $\mathrm{C} 2$ profile. Interestingly, in this orientation the butyl chains have a much broader distribution (triangles in Fig. 5 ), with a weak preference for lying at $120^{\circ}$ from the surface normal. This behavior is probably due to packing constraints on the liquid side of the interface that prevent the chain from 30 extending toward the bulk.

Having identified the preferred orientations, we can go further and estimate their relative importance by integrating the peaks of the intrinsic density profiles in Fig. 4. The results show that about $7 \%$ of IL interfacial cations are in orientation III, ${ }^{15} 16 \%$ in 35 orientation II, ${ }^{16}$ and $70 \%$ in orientation $\mathrm{I}^{17}$ The remaining $7 \%$ of molecules are in less meaningful arrangements (see, e.g., zone B of C9). Although the percentages obtained may depend on the details of the method and on the molecular model, they provide a reliable estimate of the relative significance of each orientation.

40 Experimental methods and previous simulation studies have provided only a partial view of surface IL cation orientations. SFG spectroscopy and surface tension measurements show that butyl chains are located mostly on the vapor side, but are inconclusive about the orientation of the ring. ${ }^{1}$ Conversely, direct ${ }_{45}$ recoil spectroscopy (DRS) data interpreted on the basis of a single molecular arrangement suggest that the ring is perpendicular to the surface, but the butyl chains are parallel to it. $^{13}$ Integration of our density profiles for surface atoms, considering the 3 preferred orientations shown in Fig. 6, yields 50 surface atom ratios of 2.12 for $\mathrm{H} / \mathrm{C}$ and 0.54 for $\mathrm{F} / \mathrm{C}$. Interestingly, these ratios are consistent with the DRS data. ${ }^{13}$ Finally, recent simulations have suggested the presence of both orientations I and II. ${ }^{9-12}$ With the maps obtained from the global density profiles (Fig. S2), we can confirm that orientation II is 55 predominant on the outer, convex regions of the surface, as suggested by those studies. Importantly, though, no previous simulations or experiments were able to unequivocally identify orientation III for IL cations.

In summary, we applied for the first time an intrinsic sampling ${ }_{60}$ approach to an ionic liquid, obtaining a molecular-level view of the surface with unprecedented detail. Our results suggest that experimental data should be reinterpreted considering a distribution of molecular orientations, rather than assuming a single preferred arrangement. This work helps resolve existing

${ }_{65}$ conflicts between simulations and experiments, and demonstrates the importance of intrinsic analysis techniques in the study of fluid interfaces.

\section{Acknowledgements}

The authors are grateful to the Portuguese Science and 70 Technology Foundation (FCT) and FEDER for financial support through project PTDC/EQU-FTT/104195/2008. G. H. acknowledges a research grant from FCT with the reference SFRH/BPD/73891/2010.

\section{Notes and references}

${ }_{75}{ }^{a}$ LSRE/LCM - Laboratory of Separation and Reaction Engineering, Faculdade de Engenharia da Universidade do Porto, Rua Dr. Roberto Frias, 4200-465 Porto, Portugal.Email: mjorge@fe.up.pt.

${ }^{b}$ REQUIMTE, Faculdade de Ciências da Universidade do Porto, Rua do Campo Alegre, 687, 4169-007 Porto, Portugal

$80 \dagger$ Electronic Supplementary Information (ESI) available: Technical details and complementary figures. See DOI: 10.1039/b000000x/

1 C. S. Santos, S. Baldelli, Chem. Soc. Rev., 2010, 39, 2136.

2 E. Chacón, P. Tarazona, Phys. Rev. Lett., 2003, 91, 166103.

3 J. Chowdhary, B. M. Ladanyi, J. Phys. Chem. B, 2006, 110, 15442.

854 M. Jorge, M. N. D. S. Cordeiro, J. Phys. Chem. C, 2007, 111, 17612.

5 L. B. Pártay, G. Hantal, P. Jedlovszky, A. Vincze, G. Horvai, J. Comput. Chem., 2008, 29, 945.

6 M. Jorge, P. Jedlovszky, M. N. D. S. Cordeiro, J. Phys. Chem. C, 2010, 114, 11169.

907 M. Jorge, G. Hantal, P. Jedlovszky, M. N. D. S. Cordeiro, J. Phys. Chem. C, 2010, 114, 18656.

8 J. N. Canongia Lopes, J. Deschamps, A. A. H. Pádua, J. Phys. Chem. $B, 2004, \mathbf{1 0 8}, 2038$.

9 R. M. Lynden-Bell, J. Kohanoff, M. G. del Pópolo, Faraday $95 \quad$ Discuss., 2005, 129, 57.

10 R. M. Lynden-Bell, M. G. del Pópolo, Phys. Chem. Chem. Phys., 2006, 8, 949.

11 B. L. Bhargava, S. Balasubramanian, J. Am. Chem. Soc., 2006, 128, 10073.

10012 T. Yan, S. Li, W. Jiang, X. Gao, B. Xiang, G. A. Voth, J. Phys. Chem. B, 2006, 110, 1800 .

13 G. Law, P. R. Watson, A. J. Carmichael, K. R. Seddon, Phys. Chem. Chem. Phys, 2001, 3, 2879.

14 P. Jedlovszky, A. Vincze, G. Horvai, J. Chem. Phys. 2002, 117, 1052271. 
15 Obtained by integrating the peak for zone $\mathrm{C}$ of the $\mathrm{C} 22$ profile, composed exclusively of molecules in orientation III.

16 Integrating the peak for zone A of the C9 profile (composed of molecules in orientations II and III) and subtracting our estimate for orientation III.

17 Integrating the peak for zone $\mathrm{C}$ of the $\mathrm{C} 9$ profile. 


\section{Supporting information}

\section{Technical details}

To describe molecular interactions the widely used all-atom model developed by CanongiaLopes and Pádua was applied. ${ }^{1,2} 512$ ion pairs were simulated on the NVT ensemble in a rectangular simulation box of dimensions $5 \mathrm{~nm} \times 5 \mathrm{~nm} \times 13 \mathrm{~nm}$ using the Gromacs simulation package. ${ }^{3}$ The thickness of the fluid was around $7 \mathrm{~nm}$. The temperature was kept constant at $298.15 \mathrm{~K}$ with the velocity rescaling method. ${ }^{4}$ Electrostatic interactions were truncated in real space beyond a cut-off radius of $1.5 \mathrm{~nm}$. To account for the long range part of the electrostatic interaction the PME method was used. ${ }^{5}$ Dispersion interactions were truncated by smoothly decreasing to zero between 1.1 and $1.2 \mathrm{~nm}$, with long range corrections applied beyond this region. After a $12 \mathrm{~ns}$ long equilibration run, several independent calculations were performed with a total simulation length of $80 \mathrm{~ns}$. The time step of the simulation was $2 \mathrm{fs}$. Configurations were stored at every 2 ps during the production giving in total a sample of 40000 configurations. According to the original parameterization of the potential, all $\mathrm{C}-\mathrm{H}$ bonds were kept fixed as well as the geometry of the rigid anion using the SHAKE algorithm. ${ }^{6}$

For the basis of the applied ITIM method for detecting the true set of interfacial molecules the reader is referred to the paper of Pártay et al. ${ }^{7}$ Test lines were arranged in a $100 \times 100$ grid along the $X Y$ face of the simulation cell. This test line density gives an optimal grid spacing of $0.05 \mathrm{~nm} .{ }^{8}$ The radius of the probe spheres was set to $0.2 \mathrm{~nm}$, since this value was successfully applied to several different organic phases. ${ }^{8,9}$ Note, however, that this parameter should be, in principle, optimized for each phase, but this was left for future work. One can, however, reasonably argue that the precise value of this parameter should have only a small influence on the results, as demonstrated previously. $^{7-9}$ Intrinsic properties were calculated by constructing a surface passing through all the surface atoms, based on an efficient triangular interpolation. ${ }^{10}$ We obtained symmetric profiles, 
hence all density profiles shown here are averaged over the two sides of the liquid phase. For the orientation analysis we adopted the bivariate distribution method of Jedlovszky et al. with the coordinate frame described in Figure $1 .{ }^{11}$

\section{Additional Figures}

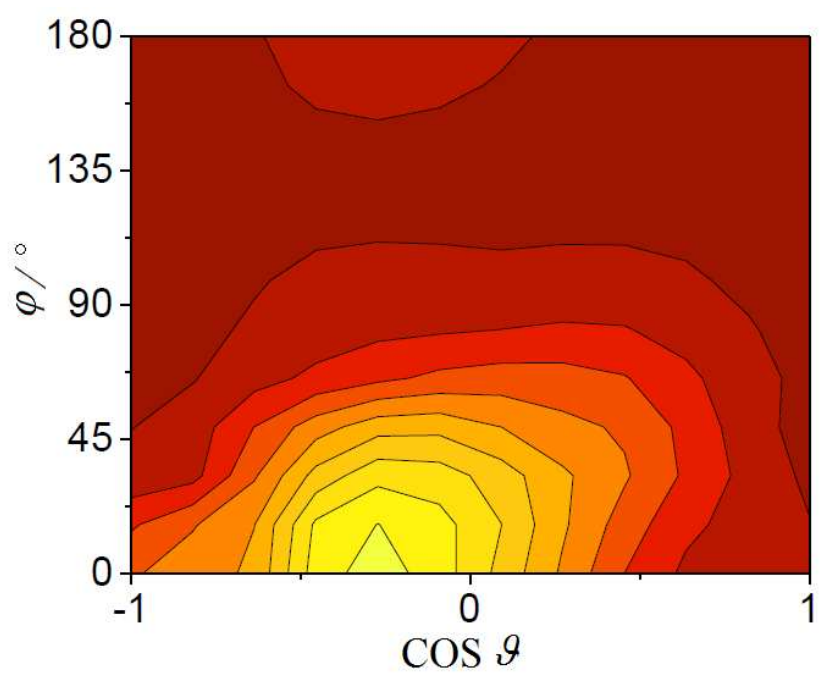

Figure S1. Orientation distribution of cation rings in the whole interfacial layer. The map is dominated by the most prevalent orientation (orientation I).

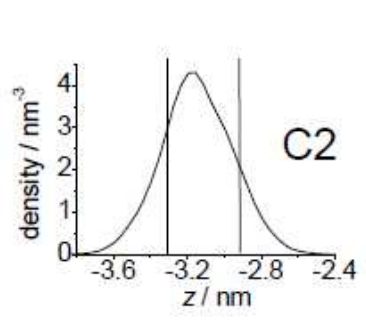

A
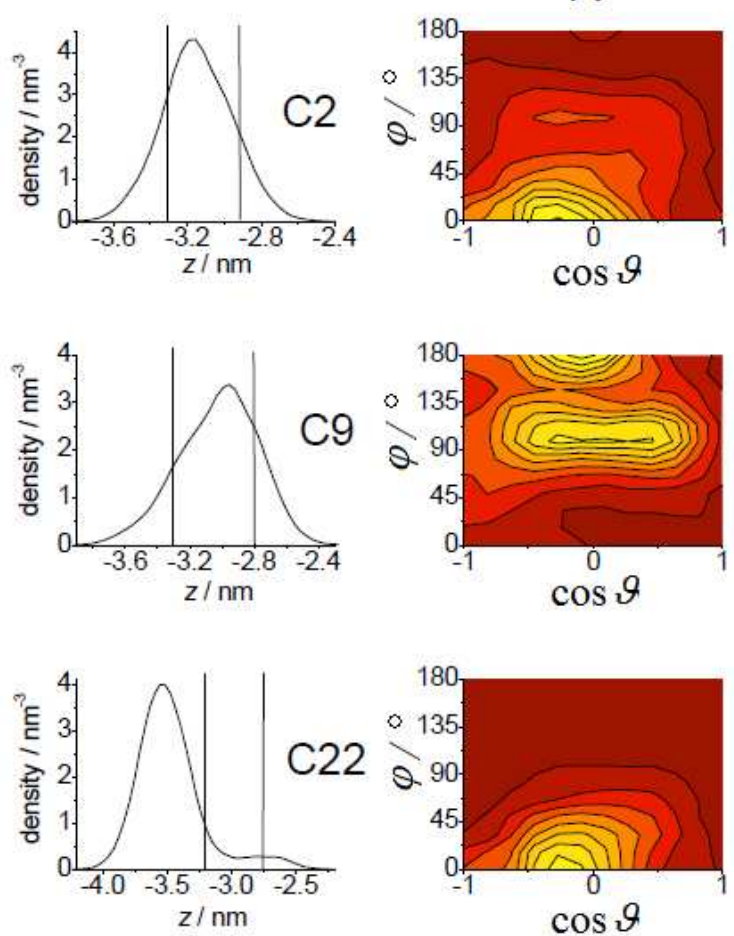
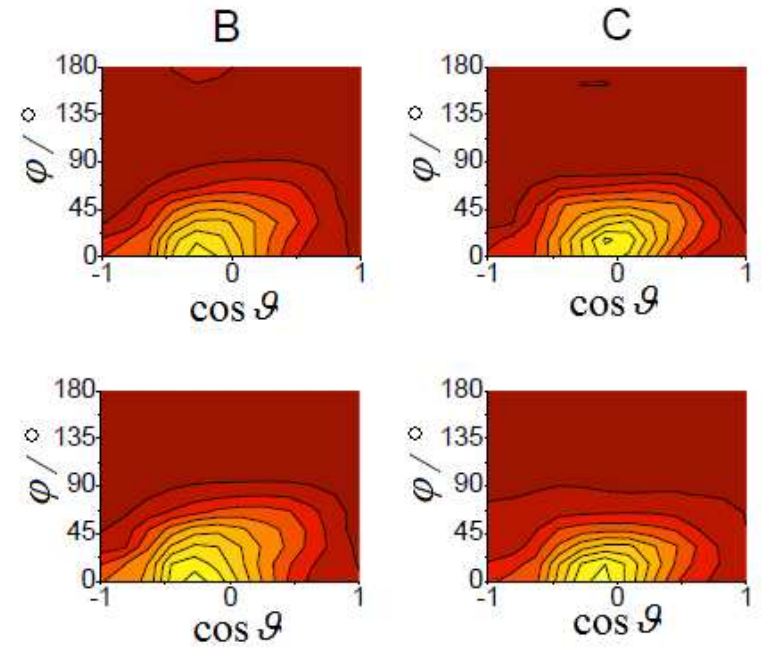
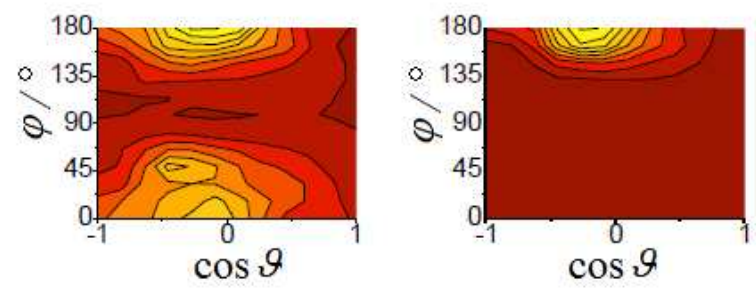
Figure S2. Orientation maps corresponding to different regions of the global density profiles. Brighter tones represent higher probabilities. Three atoms having the most structured profiles were chosen for this analysis: C2 (i.e. the carbon atom that is located symmetrically between the two nitrogen atoms), C9 (i.e. the center of the methyl group), and C22 (i.e. the terminal carbon of the butyl chain). The global distance range was divided as illustrated in the first column to highlight the most interesting orientations. Regions toward the vapor and the bulk were denoted by $A$ and $C$, respectively, while B refers to an intermediate region.

References:

(1) J. N. Canongia Lopes, J. Deschamps, A. A. H. Pádua, J. Phys. Chem. B 2004, 108, 2038.

(2) J. N. Canongia Lopes, J. Deschamps, A. A. H. Pádua, J. Phys. Chem. B 2004, 108, 11250.

(3) E. Lindhal, B. Hess, D. van der Spoel, J. Mol. Mod. 2001, 7, 306.

(4) G. Bussi, D. Donadio, M Parrinello, J. Chem. Phys. 2007, 126, 014101.

(5) U. Essman, L. Perera, M. L. Berkowitz, T. Darden, H. Lee, L. G. Pedersen, J. Chem. Phys. 1995, $103,8577$.

(6) J. P. Ryckaert, G. Ciccotti, H. J. C. Berendsen, J. Comput. Phys. 1977, 23, 327.

(7) L. B. Pártay, G. Hantal, P. Jedlovszky, A. Vincze, G. Horvai, J. Comput. Chem. 2008, $29,945$.

(8) M. Jorge, P. Jedlovszky, M. N. D. S. Cordeiro, J. Phys. Chem. C 2010, 114, 11169.

(9) G. Hantal, M. Darvas, L. B. Pártay, G. Horvai, P. Jedlovszky, J. Phys.: Condens. Matter 2010, $22,284112$.

(10) M. Jorge, G. Hantal, P. Jedlovszky, M. N. D. S. Cordeiro, J. Phys. Chem. C 2010, 114, 18656.

(11) P. Jedlovszky, A. Vincze, G. Horvai, J. Chem. Phys. 2002, 117, 2271. 